\title{
Provider Counseling and Weight Loss Outcomes in a Primary Care-Based Digital Obesity Treatment
}

\author{
Megan McVay, $P h D^{7}$, Dori Steinberg, $P h D, R D^{2,3}$, Sandy Askew, $\mathrm{MPH}^{3}$, and \\ Gary G. Bennett, $P h D^{3,4}$
}

\begin{abstract}
'Department of Health Education and Behavior, ;University of Florida, Gainesville, FL, USA; ${ }^{2}$ School of Nursing, ;Duke University, Durham, NC, USA; ${ }^{3}$ Duke Global Digital Health Science Center, Duke Global Health Institute, ;Duke University, Durham, NC, USA; ${ }^{4}$ Department of Psychology and Neuroscience, ;Duke University, Durham, NC, USA.
\end{abstract}

\begin{abstract}
BACKGROUND: Primary care-based digital health weight loss interventions offer promise for addressing obesity in underserved populations.
\end{abstract}

OBJECTIVES: To determine if primary care providers' weight counseling is associated with weight change during a weight loss intervention.

DESIGN: This is a secondary analysis of a randomized clinical trial testing a 12-month primary care-based digital health weight loss intervention.

PARTICIPANTS: Participants were community health center patients with body mass indexes of $30-44.9 \mathrm{~kg} / \mathrm{m}^{2}$.

INTERVENTIONS: The weight loss intervention included tailored behavioral goal setting; weekly goal monitoring via text messaging or interactive voice response calls; counseling calls; skills training material; and participant-tailored recommendations for provider counseling.

MAIN MEASURES: At 6 and 12 months, participants' weight was measured and they reported if their provider delivered weight counseling (general or interventionspecific) at their most recent visit and their perception of providers' empathy. Providers' documentation of weight counseling was extracted from health records.

KEY RESULTS: Participants ( $n=134-141)$ were predominantly female (70\%) and African American (55\%) with a mean age of 51 years and BMI of $36 \mathrm{~kg} / \mathrm{m}^{2}$. Participantreported provider weight counseling was not associated with weight change. However, participants whose providers documented intervention-specific counseling at any point during the intervention $(n=35)$ lost $3.1 \mathrm{~kg}$ (95\% CI 0.4 to $5.7 \mathrm{~kg}$ ) more than those whose providers documented only general weight counseling $(n=82)$ and $4.0 \mathrm{~kg}(95 \% \mathrm{CI} 0.1$ to $7.9 \mathrm{~kg})$ more than those whose providers did not document weight counseling $(n=17)$. Perceptions of provider empathy were associated with greater weight loss from 6 to 12 months $(0.8 \mathrm{~kg}$ per measure unit, $95 \%$ CI 0.07 to $1.5 \mathrm{~kg}, p=.03$ ).

CONCLUSIONS: Provider counseling that focuses specifically on engagement in a weight loss intervention may

Portions of this study were presented at the Annual Meeting of the Society for Behavioral Medicine (2017).

Electronic supplementary material The online version of this article (https://doi.org/10.1007/s11606-019-04944-5) contains supplementary material, which is available to authorized users.

Received May 14, 2018

Revised December 17, 2018

Accepted February 22, 2019

Published online March 19, 2019 enhance weight loss outcomes relative to more general weight loss advice. Counseling that enhances patients' perceptions of empathy may be most beneficial for patients' weight loss.

\section{TRIAL REGISTRATION: NCT 01827800}

KEY WORDS: weight loss; primary health care; obesity; community health centers; vulnerable populations.

J Gen Intern Med 34(6):992-8

DOI: $10.1007 /$ s11606-019-04944-5

(C) Society of General Internal Medicine 2019

\section{INTRODUCTION}

Obesity treatment guidelines recommend that healthcare providers counsel patients with obesity to lose weight. ${ }^{1}$ However, provider weight loss counseling alone - without support from a comprehensive weight loss intervention - generally produces small or no effects on weight. ${ }^{2,3}$ To achieve clinically meaningful weight outcomes, health care providers can instead refer patients to effective comprehensive weight loss interventions. ${ }^{1,4,5}$ Once patients have been referred to a weight loss intervention, health care providers are in a position to deliver opportunistic counseling encouraging engagement in the intervention and key weight loss behaviors (i.e., dietary change and physical activity). This opportunistic weight counseling could enhance weight outcomes, given that health care providers are often trusted by their patients and are perceived to have expertise. ${ }^{6-11}$ However, the effect of provider counseling on patient weight may depend on the type of the counseling. Counseling can be focused on weight generally (e.g., advice to eat less of a certain food) or more specifically on the intervention (e.g., encouragement to engage with intervention tasks). Intervention-specific counseling may have a particularly beneficial effect on weight outcomes because such counseling may increase intervention engagement. ${ }^{12}$ To our knowledge, no previous studies have examined the specific effects of weight counseling from health care providers during a weight loss intervention.

Provider communication style and provider-patient relationship quality may also relate to outcomes during a weight loss intervention conducted in a health care setting. The limited research to date on patient-provider relationship and communication characteristics and weight has been mixed. ${ }^{3,13}$ One 
study found no association between weight loss and ratings of patient-provider relationship quality. ${ }^{13}$ In another study, physicians' use of motivational interviewing techniques, such as collaborative communication, was associated with greater weight loss after a physician encounter. ${ }^{3}$

In the current study, we examined the relation between primary care providers' opportunistic weight counseling and weight change among primary care patients enrolled in a 12month trial of a digital health weight loss intervention, called Track. ${ }^{14}$ Specifically, we examined the effects of providers' general weight counseling and intervention-specific counseling as reported by participants and, separately, as documented by participants' providers in electronic health records (EHRs). We additionally examined the effects of participants' perceptions of provider empathy on weight outcomes.

\section{METHODS}

\section{Participants}

The Track trial has been described in detail elsewhere. ${ }^{14,15}$ Participants were patients at four federally qualified community health centers that were part of a community health system in central North Carolina. Participants were eligible if they had a BMI between $30.0-44.9 \mathrm{~kg} / \mathrm{m}^{2}$; weighed less than $330 \mathrm{lb}$ (weight limit for digital scale used in the intervention); had a diagnosis of hypertension, diabetes, and/or hyperlipidemia; had 2 or more visits to a participating clinic in the past year; were residents of North Carolina; could read and write in English; had a mobile phone and were willing and able to send or receive 3-9 text messages per week. Participants were excluded if they were pregnant or within 12 months postpartum; cohabitated with another study participant; were currently employed by the community health system; were participating in a similar study; had plans to move within 2 years; or had certain cardiac conditions. ${ }^{15}$ Patients with history of a condition (e.g., cancer, schizophrenia) or medication (e.g., steroids, anti-psychotics) for which weight loss is contraindicated, would affect body weight, or would impact treatment were ineligible.

Potentially eligible patients were identified via medical record review and were invited to a screening visit, followed by a baseline study visit. Eligible and consented participants $(N=351)$ were randomized to usual care or the intervention at a 1:1 ratio (see Consort Figure in Foley et al.). This trial was registered with clinicaltrials.gov (NCT 01827800). All study procedures were approved by the Duke University Institutional Review Board and the Piedmont Health Board of Advisors. Informed consent was obtained from all participants.

\section{Intervention}

Track tested a 12-month intervention which included both digital components and human support. Intervention content was informed by Social Cognitive Theory, with self-efficacy as the primary intervention target. The intervention included five components. First, each participant was assigned tailored behavior change goals from a library of obesogenic behaviors (e.g., walk 10,000 steps per day) using a computer algorithm. The behavior change goals changed every 2 months. Second, participants received a weekly interactive voice response (IVR) call or text message to report progress on their behavior change goals and providing reinforcement, motivation, and skills training tips. Third, participants received education material. Fourth, coaches (registered dietitians and two psychology graduate students) called participants 18 times over the 12-month intervention to provide additional support and skills training. Fifth, at each of participants' community health center clinic visits, providers received, via pop-up notes in electronic health records, reports on their patients' progress in the intervention, including the status of behavior change goals, weight change, and their engagement with counseling and IVR calls. These notes also included recommendations to reinforce the need for change and to endorse intervention participation. These pop-up reports occurred at every clinical visit to the health care system, regardless of the provider with which patients met. Additionally, providers received quarterly reports via email that delivered feedback on clinic-level intervention counseling rates. Primary care providers in the community health centers that participated in this study $(n=64)$ were medical doctors or nurse practitioners. Providers at these clinics did not receive any explicit instructions from the study team about documenting weight counseling. No extra clinic visits were scheduled for the purpose of this trial.

\section{Measures}

Demographic Characteristics. Participants' date of birth, gender, race, ethnicity, and highest level of education were self-reported at baseline.

Anthropometrics. Height was measured at baseline by study staff using a wall-mounted, calibrated stadiometer (Seca 222). Weight was measured by study staff at baseline, 6 month, and 12 month assessments with participants in hospital gowns using a portable electronic scale (Seca Model 876).

\section{Participant-Reported Provider Weight Communication. At 6} and 12 month assessment visits, participants who reported having a primary care appointment in the previous 6 months were asked about general weight communication by their primary care provider at their most recent appointment. Questions were developed to correspond to the 5As (Ask, Advise, Assess, Assist, and Arrange) because these are consistent with training many physicians receive for behavior change and have an evidence base in promoting smoking cessation. Derived from previously used measure, ${ }^{16}$ a single item queried about each of the following: if their "doctor" at the health care system (1) asked about weight, 
(2) advised them to lose weight, (3) assessed their readiness to lose weight, (4) referred them to a weight loss program, (5) discussed weight loss medication, (6) discussed weight loss surgery, or (7) arranged a future contact to discuss weight.

Six items were developed to assess intervention-specific communication, corresponding to aspects of the intervention that providers were encouraged to discuss with participants. Items assessed if, at their most recent appointment, providers (1) talked to them about Track; (2) asked about their progress towards Track goals; (3) encouraged them to talk to their Track coach; (4) encouraged them to take Track phone calls; (5) talked about their weight change since starting Track; or (6) provided ideas to help them meet Track goals.

Provider-Documented Weight Counseling. Provider documentation of general weight counseling or interventionspecific counseling was abstracted from EHRs. All notes in EHRs for the time frame that the participant was enrolled in the trial were reviewed by two trained staff. Ten percent of records were coded by both staff members and results compared and reconciled. Staff coded whether text referenced specified general weight counseling content, including (1) patients' physical activity, (2) patients' diet or eating behaviors, (3) a specific weight loss goal, and (4) external weight loss program. Intervention-specific counseling was coded if (1) Track was explicitly documented, (2) Track was referred to but not explicitly mentioned, (3) provider reinforced engagement in Track, or (4) Track provider update was copied into visit notes.

Provider Empathy. At 6 and 12 months, participants were asked to rate their perceptions of how empathetic and caring their provider was during their most recent appointment on the Consultation and Relational Empathy (CARE) measure. ${ }^{17}$ The CARE includes 10 items with a 1 (not at all good) to 5 (extremely good) scale. A mean of all items is computed to obtain each participant's individual score (possible range 1050). CARE has demonstrated good evidence of validity and reliability. ${ }^{17}$

\section{Data Analyses}

Developing of Counseling Groups for Participant-Reported Counseling. We aimed to form three mutually exclusive groups to reflect participants reported receipt of weight counseling at their most recent visit: (1) no counseling received, (2) general weight counseling received, or (3) intervention-specific counseling received. Participants were considered to have received intervention-specific counseling if they endorsed any of the 6 intervention-specific communication items; these items were aggregated into one variable given the very high degree of association we observed among items. Participants were considered to have received general weight counseling if they did not endorse receiving interventionspecific counseling, but endorsed any of the 7 general weight counseling items. Finally, if participants endorsed neither intervention-specific nor general weight counseling, they were considered to have received no counseling. Separate categorizations were created for the 0-6-month time frame and the 612-month time frame. Most participants who receive intervention-specific counseling also reported general weight counseling (90\% at $0-6$ months and $80 \%$ at 6-12 months).

Developing of Counseling Groups for Provider-Documented Counseling. To be consistent with the participant-reported counseling categorizations, we focused on weight counseling documentation from the medical appointment occurring immediately prior to patients' 6-month study assessment and 12month study assessment. Categorizations into counseling groups followed the same procedures as described for participant-reported counseling. We additionally created a variable that would reflect counseling documented during the entire 12 months of the intervention ("0-12 month"). The same approach to categorization into counseling groups was used as described above; however, all progress notes in the health care system health records during the 12-month intervention were considered.

Statistical Analyses. We present here results for the intervention arm participants only; results for the usual care arm are presented in the online appendix. Separate models were run for each time period of interest (0-6, 6-12, and for provider-documented counseling, 0-12 months). For participant-reported counseling, participants who reported that they did not report having a medical visit during the time period of interest were excluded. For provider-documented weight counseling, analyses excluded those who did not give permission to abstract their data at either time point, who became ineligible over the course of the study, or who did not have an appointment recorded during the time period of interest. The majority of participants had both participantreported and provider-documented data (80\% for $0-$ 6 months and $77 \%$ for 6-12 months). To determine the relationship between provider counseling and weight, we generated mixed models using weight as the outcome and a time by counseling group interaction term as the predictor of interest. The models used an unstructured covariance matrix. Missing data were addressed with the intent to treat methods using maximum likelihoods. To determine the impact of perceived provider empathy on weight, we used a similar modeling strategy featuring the continuous CARE score by time interaction as the term of interest. We also generated a set of models adjusting for age, gender, race, education, clinic site, length of time between last clinical appointment and assessment, and total number of clinic visits during the intervention (see Online Appendix for descriptive data on time between last clinical appointment and assessment, and total number of clinic visits 
during the intervention). However, these adjustments did not improve the models or meaningfully alter the estimates; thus, only unadjusted models are presented here.

\section{RESULTS}

Participant characteristics are presented in Table 1. Figure 1 shows the portion of participants who reported weight counseling and who had provider documentation of counseling.

Participant report of provider weight counseling was not associated with weight change at $0-6$ or $6-12$ months (see Table 2). Provider-documented weight counseling from $0-6$ to 6-12 months was not associated with weight change (see Table 2). However, there was a significant association between provider-documented counseling across all 0-12-month appointments and weight change; participants documented to have received intervention-specific counseling lost $4.0 \mathrm{~kg}$ more weight than those who were documented to have received no counseling and $3.1 \mathrm{~kg}$ more than those who received general weight counseling. As a post hoc follow-up, we examined associations between provider-documented counseling during the $0-12$-month time frame and engagement in intervention counseling calls and self-monitoring and found no association (see online Appendix).

Table 1 Baseline Characteristics of Study Participants Included in Participant-Reported and Provider-Documented Analyses

\begin{tabular}{|c|c|c|}
\hline & $\begin{array}{l}\text { Participant- } \\
\text { reported } \\
\text { analytic sample } \\
(n=141)\end{array}$ & $\begin{array}{l}\text { Provider- } \\
\text { documented } \\
\text { analytic sample } \\
(n=134)\end{array}$ \\
\hline Age, years, $M(\mathrm{SD})$ & $51.4(8.9)$ & $50.9(9.0)$ \\
\hline Weight, $\mathrm{kg}, M(\mathrm{SD})$ & $98.7(14.2)$ & $98.9(14.0)$ \\
\hline BMI $\left(\mathrm{kg} / \mathrm{m}^{2}\right)$ & $36.0(4.1)$ & $35.9(4.1)$ \\
\hline \multicolumn{3}{|l|}{ Sex, $n(\%)$} \\
\hline Women & $99(70 \%)$ & $93(69 \%)$ \\
\hline Men & $42(30 \%)$ & $41(31 \%)$ \\
\hline \multicolumn{3}{|l|}{ Education, $n(\%)$} \\
\hline Less than HS degree & $18(13 \%)$ & $18(13 \%)$ \\
\hline HS graduate & $54(38 \%)$ & $51(38 \%)$ \\
\hline Some college or & $58(41 \%)$ & $56(42 \%)$ \\
\hline associate's degree & & \\
\hline $\begin{array}{l}4 \text { year college degree } \\
\text { or higher }\end{array}$ & $11(8 \%)$ & $9(7 \%)$ \\
\hline \multicolumn{3}{|l|}{ Race/ethnicity, $n(\%)$} \\
\hline Non-Hispanic Black & $79(56 \%)$ & $73(54 \%)$ \\
\hline Non-Hispanic White & $38(27 \%)$ & $37(28 \%)$ \\
\hline Hispanic & $17(12 \%)$ & $18(13 \%)$ \\
\hline Non-Hispanic Other & $7(5 \%)$ & $6(4 \%)$ \\
\hline \multicolumn{3}{|l|}{ Marital Status, $n(\%)$} \\
\hline $\begin{array}{l}\text { Not married or living } \\
\text { with partner }\end{array}$ & $74(52 \%)$ & $69(51 \%)$ \\
\hline $\begin{array}{l}\text { Married or living with } \\
\text { partner }\end{array}$ & $67(48 \%)$ & $65(49 \%)$ \\
\hline
\end{tabular}

Participant-reported analytic sample includes all participants who reported having a visit with their health care center provider during the prior 6 months at either the 6-month or 12-month assessment. Provider-documented analytic sample includes all participants who had an available medical visit note during the entire 12-month intervention
CARE scores were $M=42.1(\mathrm{SD}=9.9)$ and $M=41.1$ ( $\mathrm{SD}=$ $10.0)$ at 6 and 12 months, respectively. Greater ratings of provider empathy were associated with more weight loss, and this relationship was statistically significant during the 6-12-month time frame. Specifically, a 10-unit increase in reported CARE score was associated with a mean weight change of $-0.9 \mathrm{~kg}(95 \% \mathrm{CI}-2.0$ to $0.2 \mathrm{~kg}, p=.12)$ from 0 to 6 months and $-0.8 \mathrm{~kg}(95 \% \mathrm{CI}-1.5$ to $-0.07 \mathrm{~kg}, p=.03)$ from 6 to 12 months.

\section{DISCUSSION}

Past studies have shown that primary care provider weight loss counseling in the absence of a weight loss intervention can lead to an increase in efforts to lose weight but does not meaningfully affect weight. ${ }^{2}$ Primary care providers can assist patients in losing weight by referring them to a comprehensive weight loss intervention. ${ }^{1,4,5}$ However, it has been unclear to what extent opportunistic weight counseling by providers during a weight loss intervention could influence weight outcomes. In the current study, we examined associations between primary care providers' counseling and weight change among community health center patients enrolled in a digital health weight loss intervention. We found that patients' self-report of weight counseling from their provider was not associated with weight outcomes. However, patients whose providers' documented intervention-specific counseling at any point during the intervention lost 3-4 kg more than patients who had no weight counseling or only general weight counseling.

The observed association between provider documentation of intervention counseling and weight loss suggests that a patient-provider discussion about continued engagement with an intervention may be influential during a weight loss intervention. This is consistent with the supportive accountability model, which suggests that human contact during a digital health intervention will lead to greater intervention engagement, particularly when support individuals are viewed as trustworthy and legitimate. ${ }^{12}$ However, previous research examining the effects of different levels of (non-physician) human support on intervention engagement and outcomes during weight-focused digital health interventions has been mixed. ${ }^{18-}$ ${ }^{20}$ Potentially, the perceived legitimacy of health care providers and the bond that some patients experience with their providers may contribute to the impact of provider counseling. ${ }^{12}$ Replication of these findings and experimental approaches to studying this relationship are warranted. Combined with previous findings, ${ }^{15}$ these results suggest that clinicians should advise their patients to join evidence-based weight loss programs and should provide support and encouragement to engage in the intervention at each subsequent clinical encounter.

We did not observe a direct relationship between providerdocumented intervention-specific weight counseling and engagement with intervention counseling calls or self- 
a

0-6 months

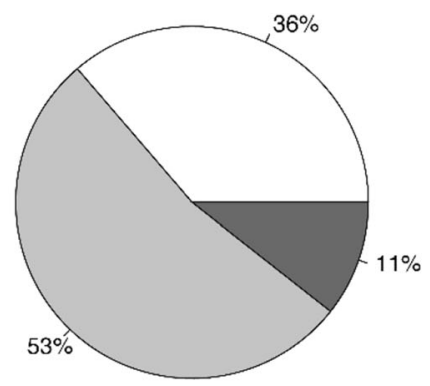

b

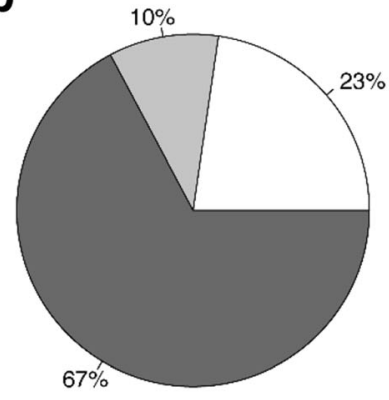

0-6 months
6-12 months
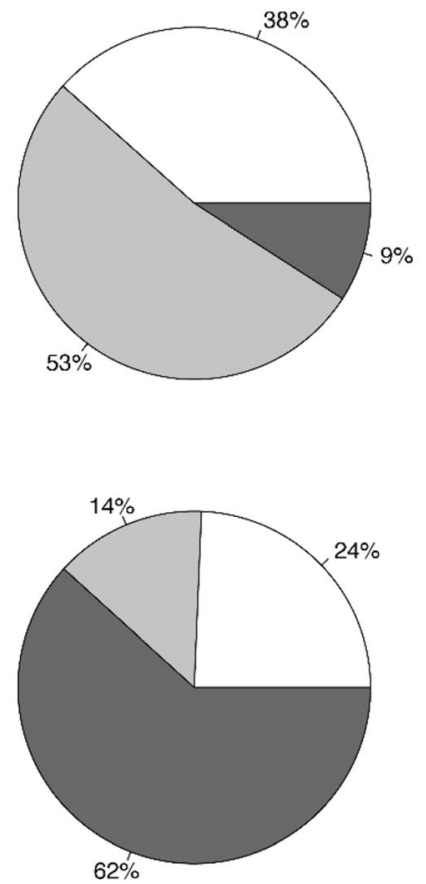

6-12 months
0-12 months
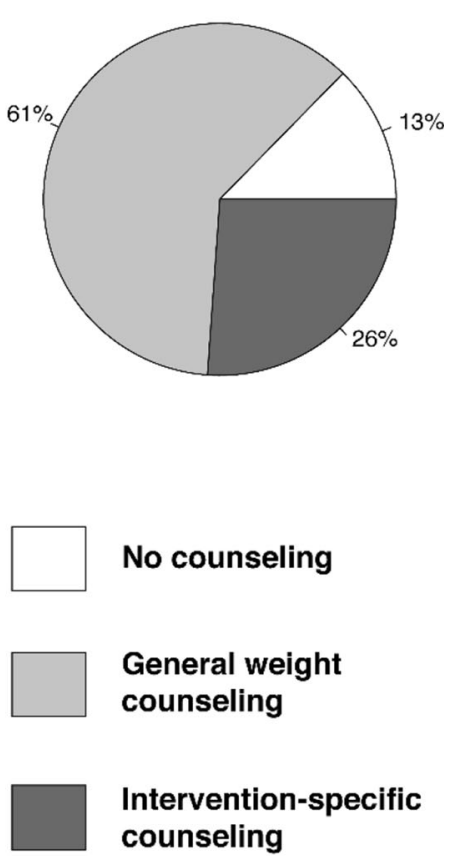

No counseling

General weight counseling

Intervention-specific counseling

Fig. 1 Frequency of intervention-specific counseling, general weight counseling, and no counseling at 0-6 months and 6-12 months for intervention arm participants. Panel $\mathbf{A}$ is frequencies for provider-documented counseling and panel $\mathrm{B}$ is frequencies for participant-reported counseling.

monitoring, suggesting that these forms of intervention engagement were unlikely to account for the observed relationship. It is possible that individuals who received interventionspecific counseling become more engaged with the intervention in ways that are not captured with these measures, for example, becoming more active in coaching call discussions or more adherent to behavior change goals.
The frequency of provider-documented and participantreported counseling differed substantially. This could be related to providers not documenting discussions that were had with patients about the intervention or to patients' errors in reporting conversations. We can speculate that differences in the relationship between these two variables and weight could be due to provider documentation of weight counseling

Table 2 Participant-Reported and Provider-Documented Counseling Groups and Unadjusted Weight Change Among Intervention Arm Participants

\begin{tabular}{|c|c|c|c|c|c|c|}
\hline & & $n$ & $\begin{array}{l}\text { Estimated within-group weight } \\
\text { change, } \mathrm{kg}, M \text { (SD) }\end{array}$ & $\begin{array}{l}\text { Estimated between-group weight } \\
\text { difference, } \mathrm{kg}, M \text { (SD) }\end{array}$ & $p$ & Type-3p \\
\hline \multicolumn{7}{|c|}{ Participant-reported counseling } \\
\hline \multirow[t]{3}{*}{$0-6$ months } & No counseling & 27 & $-3.8(-6.2$ to -1.5$)$ & $0.3(-2.4$ to 3.0$)$ & 0.84 & 0.95 \\
\hline & General weight counseling & 12 & $-3.6(-7.1$ to -0.1$)$ & $0.5(-3.2$ to 4.3$)$ & 0.78 & \\
\hline & Intervention-specific counseling & 80 & $-4.1(-5.5$ to -2.7$)$ & Ref & Ref & \\
\hline \multirow[t]{3}{*}{$6-12$ months } & No counseling & 28 & $0.3(-1.2$ to 1.7$)$ & $0.4(-1.4$ to 2.1$)$ & 0.68 & 0.76 \\
\hline & General weight counseling & 16 & $-0.6(-2.6$ to 1.3$)$ & $-0.6(-2.7$ to 1.6$)$ & 0.60 & \\
\hline & Intervention-specific counseling & 71 & $-0.1(-1.0$ to 0.8$)$ & Ref & Ref & \\
\hline \multicolumn{7}{|c|}{ Provider-documented counseling } \\
\hline \multirow[t]{3}{*}{$0-6$ months } & No counseling & 41 & $-4.0(-5.8$ to -2.2$)$ & $1.8(-2.1$ to 5.7$)$ & 0.36 & 0.54 \\
\hline & General weight counseling & 60 & $-3.7(-5.2$ to -2.2$)$ & $2.1(-1.6$ to 5.8$)$ & 0.27 & \\
\hline & Intervention-specific counseling & 12 & $-5.8(-9.2$ to -2.4$)$ & Ref & Ref & \\
\hline \multirow{3}{*}{ 6-12 months } & No counseling & 38 & $-0.4(-1.7$ to 0.8$)$ & $-0.2(-3.0$ to 2.6$)$ & 0.91 & 0.72 \\
\hline & General weight counseling & 52 & $0.2(-0.8$ to 1.3$)$ & $0.5(-2.2$ to 3.2$)$ & 0.71 & \\
\hline & Intervention-specific counseling & 9 & $-0.3(-2.8$ to 2.2$)$ & Ref & Ref & \\
\hline \multirow{3}{*}{ 0-12 months } & No counseling & 17 & $-2.5(-5.7$ to 0.7$)$ & $4.0(0.1$ to 7.9$)$ & 0.04 & 0.04 \\
\hline & General weight counseling & 82 & $-3.4(-4.9$ to -2.0$)$ & $3.1(0.4$ to 5.7$)$ & 0.02 & \\
\hline & Intervention-specific counseling & 35 & $-6.5(-8.7$ to -4.3$)$ & Ref & & \\
\hline
\end{tabular}


reflecting longer or more in-depth intervention-specific counseling than occurred when participants reported counseling.

Our finding that participants' ratings of their providers' empathy were associated with greater weight loss is consistent with some previous research, ${ }^{3,21}$ though other studies have reported a lack of association with similar constructs. ${ }^{13}$ Training in communication styles that enhance patient perceptions of provider empathy may be beneficial for enhancing weight loss and other outcomes among primary care patients.

Several limitations should be noted. Although Track was a randomized controlled trial, the counseling groups in this secondary analysis were not randomly assigned and we cannot conclude a causal relationship between counseling and weight. It is possible that patients or providers are more likely to bring up the intervention when the participants are already having greater success with weight loss; a bidirectional relationship is also plausible. Another limitation of this study is that we did not have objective data (e.g., audio-recordings) on patientprovider interactions nor do we have extensive data on the medical visit (e.g., the length of the visit). Participants may not have accurately remembered the nature of their clinic visit and providers may have selectively reported or misreported their counseling. If these inaccuracies were systematic, this could bias the results. Another limitation is that numerous analyses were conducted for this report, increasing the chance of a type I error, and that there were a small number of observations for some cells; replication is warranted. This study also has several strengths, including that this association was examined in a low-income, socially disadvantaged population and the ability to examine these relationships with multiple measures of provider/patient interaction (participant-reported and provider-documented).

In summary, participants in a primary care-based, digital health weight loss intervention whose primary care providers' document intervention-specific counseling may be more successful at weight loss. This suggests that providing access to digital health weight loss programs in a primary care setting, alongside efforts to enhance providers' intervention-specific counseling, may contribute to improved weight management among underserved populations. Efforts to enhance a provider's ability to communicate empathy and concern may also result in greater weight loss. Future research using an experimental design is warranted to confirm the role of intervention-specific provider weight counseling on weight outcomes.

Acknowledgements: We wish to acknowledge the Track study participants and the health care providers for making this research possible. We also acknowledge the research assistants who assisted with data collection on EHR provider counseling data, Jacob Christy and Jasmine Burroughs.

Corresponding Author: Megan McVay, PhD; Department of Health Education and Behavior, University of Florida, Gainesville, FL, USA (e-mail: Megan.mcvay@ufl.edu).
Funding Information This research is funded in part by the following grants: R01DK093829 to Dr. Gary Bennett; K23HL127334 to Dr. Megan McVay; K12HD043446 to Dr. Dori Steinberg.

\section{Compliance with Ethical Standards:}

Conflict of Interest: Gary Bennett holds equity in Coeus Health, LLC, and serves on the scientific advisory board of Nutrisystem, is a member of the board of directors at Girl Trek, and is a past president and a member of the board of directors at the Society of Behavioral Medicine. All remaining authors declare that they do not have a conflict of interest.

\section{REFERENCES}

1. LeBlanc E, O'Connor E, Whitlock EP, Patnode C, Kapka T. Screening for and Management of Obesity and Overweight in Adults. Rockville (MD): Agency for Healthcare Research and Quality (US); 2011.

2. Rose SA, Poynter PS, Anderson JW, Noar SM, Conigliaro J. Physician weight loss advice and patient weight loss behavior change: a literature review and meta-analysis of survey data. Int J Obes. 2013;37(1):118-28.

3. Pollak KI, Alexander SC, Coffman CJ, Tulsky JA, Lyna P, Dolor RJ, et al. Physician communication techniques and weight loss in adults. Am J Prev Med. 2010;39(4):321-8.

4. Jebb SA, Ahern AL, Olson AD, Aston LM, Holzapfel C, Stoll J, et al. Primary care referral to a commercial provider for weight loss treatment versus standard care: a randomised controlled trial. Lancet. 2011; 378(9801):1485-92.

5. Aveyard P, Lewis A, Tearne S, Hood K, Christian-Brown A, Adab P, et al. Screening and brief intervention for obesity in primary care: a parallel, two-arm, randomised trial. Lancet. 2016 388(10059):2492-500.

6. Gallagher TH, St. Peter RF, Chesney M, Lo B. Patients' attitudes toward cost control bonuses for managed care physicians. Health Aff. 2001;20(2):186-92.

7. Lake T. Do HMOs make a difference? Consumer assessments of health care. Inquiry. 1999; 36(4):411-8.

8. Anderson LA, Dedrick RF. Development of the Trust in Physician Scale: A Measure to Assess Interpersonal Trust in Patient-Physician Relationships. Psychol Rep. 1990; 67(3_suppl):1091-100.

9. Kao AC, Green DC, Davis NA, Koplan JP, Cleary PD. Patients' trust in their physicians. J Gen Intern Med. 1998; 13(10):681-6.

10. Moreau A, Carol L, Dedianne MC, Dupraz C, Perdrix C, Lainé X, et al. What perceptions do patients have of decision making (DM)? Toward an integrative patient-centered care model. A qualitative study using focusgroup interviews. Patient Educ Couns. 2012; 87(2):206-11.

11. Lupton D. Consumerism, reflexivity and the medical encounter. Soc Sci Med. 1997; 45(3):373-81.

12. Mohr DC, Cuijpers P, Lehman K. Supportive Accountability: A Model for Providing Human Support to Enhance Adherence to eHealth Interventions. J Med Internet Res [Internet]. 2011 Mar 10 [cited 2018 May 4];13(1).

13. Bennett WL, Wang N-Y, Gudzune KA, Dalcin AT, Bleich SN, Appel LJ, et al. Satisfaction with primary care provider involvement is associated with greater weight loss: Results from the practice-based POWER trial. Patient Educ Couns. 2015; 98(9):1099-105.

14. Foley P, Steinberg D, Levine E, Askew S, Batch BC, Puleo EM, et al. Track: A randomized controlled trial of a digital health obesity treatment intervention for medically vulnerable primary care patients. Contemp Clin Trials. 2016; 48:12-20.

15. Bennett GG, Steinberg D, Askew S, Levine E, Foley P, Batch BC, Svetkey LP, Bosworth HB, Puleo EM, Brewer A, DeVries A. Effectiveness of an app and provider counseling for obesity treatment in primary care. Am J Prev Med. 2018.

16. Bodner ME, Dolor RJ, Ǿstbye T, Lyna P, Alexander SC, Tulsky JA, et al. Accuracy and congruence of patient and physician weight-related discussions from project CHAT (Communicating Health: Analyzing Talk). J Am Board Fam Med. 2014; 27(1):70-7.

17. Mercer SW, Maxwell M, Heaney D, Watt GC. The consultation and relational empathy (CARE) measure: development and preliminary validation and reliability of an empathy-based consultation process measure. Fam Pract. 2004; 21(6):699-705.

18. Glasgow RE, Kurz D, King D, Dickman JM, Faber AJ, Halterman E, et al. Outcomes of minimal and moderate support versions of an internet- 
based diabetes self-management support program. J Gen Intern Med. 2010; 25(12):1315-22.

19. Dennison L, Morrison L, Lloyd S, Phillips D, Stuart B, Williams S, et al. Does brief telephone support improve engagement with a web-based weight management intervention? Randomized controlled trial. J Med Internet Res. 2014 Mar 28.

20. Harvey-Berino J, West D, Krukowski R, Prewitt E, VanBiervliet A, Ashikaga T, et al. Internet delivered behavioral obesity treatment. Prev Med. 2010; 51(2):123-8.
21. McVay MA, Yancy WS, Scott CN, Wilson-Barlow L, Woolson S, McSherry WC, et al. Patient factors associated with initiation of behavioral weight loss treatment: a prospective observational study in an integrated care setting. Transl Behav Med. 2017;7(1):75-83.

Publisher's Note: Springer Nature remains neutral with regard to jurisdictional claims in published maps and institutional affiliations. 